\title{
THE NON-UNICITY OF THE FILM THICKNESS IN THE HYDRODYNAMIC LUBRICATION: NOVEL APPROACH GENERATING EQUIVALENT MICRO-GROOVES AND ROUGHNESS
}

\author{
M. EL GADARI* \\ University Moulay Ismail, ENSAM, Meknes, MOROCCO \\ E-mail: m.elgadari@ensam-umi.ac.ma \\ M. HAJJAM \\ Department D3, Prime Institute, UPR3346, University of Poitiers, FRANCE
}

\begin{abstract}
Since the 1960s, all studies have assumed that a film thickness " $h$ " provides a unique pressure field " $p$ " by resolving the Reynolds equation. However, it is relevant to investigate the film thickness unicity under a given hydrodynamic pressure within the inverse theory. This paper presents a new approach to deduce from an initial film thickness a widespread number of thicknesses providing the same hydrodynamic pressure under a specific condition of gradient pressure. For this purpose, three steps were presented: 1) computing the hydrodynamic pressure from an initial film thickness by resolving the Reynolds equation with Gümbel's cavitation model, 2) using a new algorithm to generate a second film thickness, 3) comparing and validating the hydrodynamic pressure produced by both thicknesses with the modified Reynolds equation. Throughout three surface finishes: the macroshaped, micro-textured, and rough surfaces, it has been demonstrated that under a specific hydrodynamic pressure gradient, several film thicknesses could generate the same pressure field with a slight difference by considering cavitation. Besides, this paper confirms also that with different ratios of the averaged film thickness to the root mean square (RMS) similar hydrodynamic pressure could be generated, thereby the deficiency of this ratio to define the lubrication regime as commonly known from Patir and Cheng theory.
\end{abstract}

Key words: Reynolds equation, inverse theory, macro-shape, micro-texture, cavitation, lifting force, friction force, flow rate.

\section{Introduction}

The differential equation that governs the generation of pressure in lubricating films is known as the Reynolds equation. In steady-state condition, this equation is given for one dimensional, isoviscous, and incompressible hydrodynamic lubrication problem by:

$$
\frac{d}{d x}\left(\frac{h^{3}}{6 \mu U} \frac{d p}{d x}\right)=\frac{d h}{d x}
$$

where: $x$ is the axial direction, $U$ is the velocity of the lower surface, and $\mu$ is the lubricant viscosity.

To design the lubricated components (bearings, thrust, seals ...) Eq.(1.1) is solved by using two theories:

- The first, so called direct approach, is performed to dimension the supporting and/or guiding devices [1-3]. For a given film thickness, $h(x)$ the pressure distribution, $p(x)$ within the fluid film is obtained by solving the Reynolds equation (1.1).

\footnotetext{
${ }^{*}$ To whom correspondence should be addressed
} 
- The other is the inverse method that is widely used for elastomeric seals. Indeed, according to this theory, the static contact pressure $p s(x)$ is assumed equal to the hydrodynamic pressure $p(x)$ and the film thickness $h(x)$ is computed by using the first and second derivatives of $p(x)$ [4-6] under non-cavitation conditions.

This method is based on the Reynolds equation and two computational procedures are possible (1.2) and (1.3):

$$
\begin{aligned}
& \frac{h^{3}}{6 \mu U} \frac{d p}{d x}=h-h^{*}, \\
& \frac{d h}{d x}\left\{\frac{h^{2}}{2 \mu U} \frac{d p}{d x}-1\right\}+\frac{h^{3}}{6 \mu U} \frac{d^{2} p}{d x^{2}}=0 .
\end{aligned}
$$

Usually, Eq.(1.2) is most frequently used in the inverse hydrodynamic lubrication [6] to determine $h(x)$ where $h^{*}$ is the film thickness at the location of the maximum pressure. The other approach is rarely used and is given by Eq.(1.3) with first and second derivative pressures $d p / d x$ and $d^{2} p / d x^{2}$.

Additionally, when the surface roughness is excessive, two approaches are used to resolve the Reynolds equation:

- Stochastic method, so-called Patir and Cheng flow factors method [7] by solving the transformed (or averaged) Reynolds equation for micro bearings having a realistic surface roughness.

- Deterministic method by assuming the rough surface as an analytical function. In most cases, it is given by sinusoidal form [8].

The proposed method describes a new approach to find different geometries, micro-grooves and roughnesses of the lubricated contact that keep the same operational parameters: friction force $F$, flow rate $Q$ and lifting force $W$.

$$
\begin{aligned}
& W=\int_{0}^{L} p d x, \\
& F=\int_{0}^{L}-\left(\frac{\mu U}{h}+\frac{h}{2} \frac{d p}{d x}\right) d x, \\
& Q=\frac{-h^{3}}{12 \mu} \frac{d p}{d x}+\frac{U h}{2} .
\end{aligned}
$$

To consider the cavitation effect, the most frequently used methods are:

- Method 1: In 1921, Gümbel [11] proposed simply to neglect pressures less than atmospheric pressure. Thus, to compute the hydrodynamic pressure in a full film lubricated contact, the Reynolds equation (1.1) is resolved with the cavitation condition:

$$
p=p_{c a v} \quad \text { where } \quad p \leq p_{a t m} .
$$

- Method 2: The following modified Reynolds equation [12] is resolved: 


$$
\frac{d}{d x}\left(h^{3} \frac{d \phi D}{d x}\right)=6 \mu U\left(\frac{d h}{d x}+\frac{d(1-\phi) D}{d x}\right)
$$

where $\phi$ is the cavitation index equal to 0 in the cavitation zone when $D \leq 0$ and equal to 1 in the active zone when $D>0$.

By considering the replenishment $r$, where $\rho$ and $\rho_{0}$ are the density of the cavitated zone and lubricant, respectively:

$$
r=\frac{\rho}{\rho_{0}} h
$$

We admit also:

$$
\begin{aligned}
& p=D \quad \text { and } \quad r=h, \quad \text { when } \quad \phi=1, \\
& p=p_{c a v} \quad \text { and } \quad r=D+h, \quad \text { else. }
\end{aligned}
$$

Before beginning the numerical analysis, the reader must keep in mind the processing steps:

- First analysis: with an initial film thickness $H_{1}(x)$, the Reynolds equation (1.1) is resolved, and the hydrodynamic pressure $p(x)$ with cavitation effect is searched according to Gümbel [11] condition.

- Second analysis: based on the hydrodynamic pressure $p(x)$ of the first analysis an original approach is proposed to compute all the different film thicknesses $\mathrm{H}_{2}(x)$.

- Third analysis: by replacing the two film thicknesses $H_{1}(x)$ and $H_{2}(x)$ in the modified Reynolds equation (1.6), the hydrodynamic pressure for each film thickness is computed to compare and discuss the accuracy of the approach.

\section{Numerical approach}

\subsection{Computing the hydrodynamic pressure}

The proposed approach consists of two steps depending on each other:

- Step 1: By using an appropriate numerical method (finite differences, finite volumes, and finite elements, ...), the Reynolds equation (1.1) corresponding to the first analysis and modified Reynolds equation (1.6) for the third analysis is resolved. Thus, according to the computed pressure $p(x)$, the lifting force $W$, the friction force $F$ and the flow rate $Q$ are deduced from Eqs (1.4).



Fig.1. Schematic representation of the Finite Difference Approach. 
In this initial step, the film thickness $h(x)$ is assumed known and equal to the guessed initial thickness $H_{l}(x)$. Equations (1.1) and (1.6) are discretized with the classical finite volumes method briefly described in Fig.1. Thus the discretized equations are given by:

$$
A_{p} p_{p}+A_{O} p_{O}+A_{E} p_{E}+S_{c}=0
$$

where for the Reynolds equation (1.1) with the Gümbel [11] condition for the cavitation effect:

$$
\begin{aligned}
& A_{P}=-\frac{h_{o}^{3}+h_{e}^{3}}{\Delta x}, \\
& A_{O}=\frac{h_{o}^{3}}{\Delta x}, \\
& A_{E}=\frac{h_{e}^{3}}{\Delta x}, \\
& S c=-6 \mu U\left(h_{e}-h_{o}\right)
\end{aligned}
$$

and for the modified Reynolds equation (1.6):

$$
\begin{aligned}
& A_{P}=-2 \frac{h_{O}^{3}+h_{E}^{3}+2 h_{P}^{3}}{\Delta x} \phi_{P}-6 \mu U\left(1-\phi_{P}\right), \\
& A_{O}=2 \frac{h_{O}^{3}+h_{P}^{3}}{\Delta x} \phi_{O}+6 \mu U\left(1-\phi_{O}\right), \\
& A_{E}=2 \frac{h_{E}^{3}+h_{P}^{3}}{\Delta x} \phi_{E}, \\
& S c=-6 \mu U\left(h_{e}-h_{o}\right) .
\end{aligned}
$$

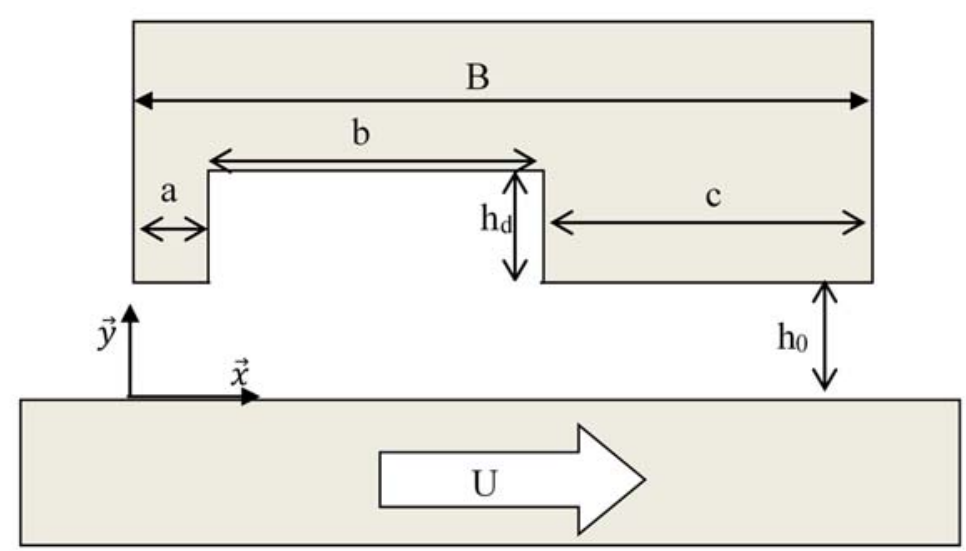

Fig.2. Schematic representation of the textured pad [9]. 
To validate the numerical model used in our hydrodynamic lubrication simulations, we refer to the work of Fowell et al. $[9,10]$ that studied the effects of textured surfaces. The analytical formulas proposed in this work allow simple but at the same time precise calculation of the pressure distribution across a textured surface. Cavitation zones are also located in the contact using a formulation based on mass conservation.

The geometry proposed by Fowell [9] in Fig.2. consists of a slot located on the stationary surface of a parallel faced pad. Thereby, in the divergent-convergent zone with the operating conditions (Tab.1.), cavitation could occur.

Table 1. Geometry and operating conditions of the textured pad [9].

\begin{tabular}{|l|l|}
\hline Parameter & Numerical value \\
\hline Pad length & $B=20 \mathrm{~mm}$ \\
\hline Inlet length & $a=4 \mathrm{~mm}$ \\
\hline Slot length & $b=6 \mathrm{~mm}$ \\
\hline Outlet length & $c=10 \mathrm{~mm}$ \\
\hline Minimum film thickness & $h_{0}=1 \mathrm{micron}$ \\
\hline Depth of pocket & $h_{d}=5 \mathrm{micron}$ \\
\hline Velocity & $U=1 \mathrm{~m} / \mathrm{s}$ \\
\hline Dynamic viscosity & $m=0.01 \mathrm{~Pa} . \mathrm{s}$ \\
\hline Ambient pressure & $p_{0}=0.1 \mathrm{MPa}$ \\
\hline Cavitation pressure & $p_{c a v}=0 \mathrm{MPa}$ \\
\hline
\end{tabular}

The boundary conditions are given by: at $x=0, p(0)=p_{0}$ and at $x=B, p(B)=p_{0}$.

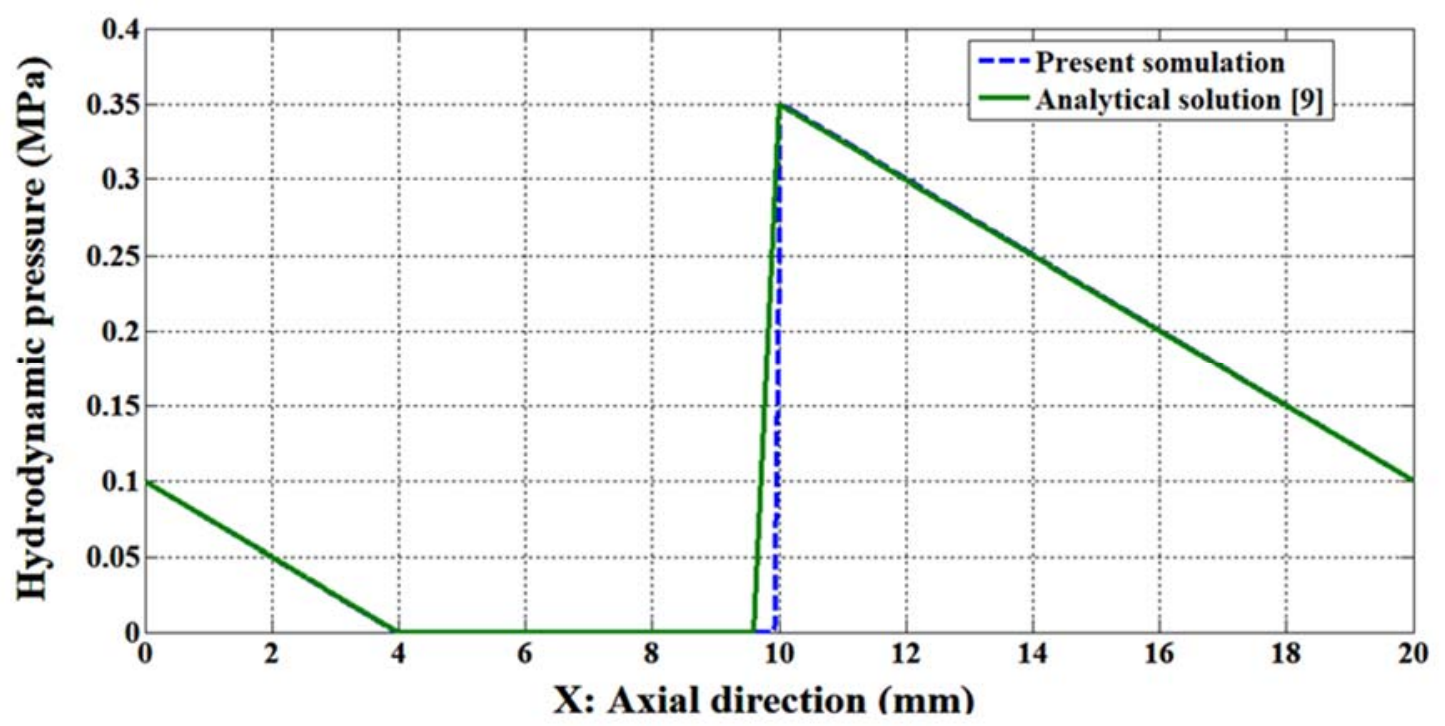

Fig.3. Comparison of the present model and results of [9].

Comparing simulations of the present model and the analytical results [9], Fig.3. confirms a good agreement between the pressure distributions. However, the figure shows that the difference is mainly caused 
by the discontinuous domain of the film thickness $h(x)$. Indeed, the large mesh size of the geometry impacts the derivative accuracy of the function $h(x)$.

\subsection{Computing different film thickness distributions}

A single and unique film thickness investigation can now be initiated to generate the same hydrodynamic pressure given by the Reynolds equation solution. Let us assume at the beginning the existence of two roots for Eq.(1.2), $H_{1}$ and $H_{2}$. Thus Eq.(1.2) becomes:

$$
\begin{aligned}
& \frac{H_{1}^{3}}{6 \mu U} \frac{d p}{d x}=H_{1}-h^{*}, \\
& \frac{H_{2}{ }^{3}}{6 \mu U} \frac{d p}{d x}=H_{2}-h^{*}
\end{aligned}
$$

where: $H_{l}(x)$ is equal to the film thickness, $h(x)$ is initially given.

In this section, we aim to check if $H_{2}(x)$ is equal to $H_{l}(x)$ whatever $x$ between 0 and $L$. By subtracting Eqs (2.4) from (2.3), we find:

$$
\left(H_{2}^{3}-H_{1}^{3}\right) \frac{d p}{d x}-6 \mu U\left(H_{2}-H_{1}\right)=0
$$

Thus the equivalent equation with $H_{2}$ as an unknown thickness is given by:

$$
\left(H_{2}^{2}+H_{1} H_{2}+H_{1}^{2}\right) \frac{d p}{d x}-6 \mu U=0 \quad \text { where } \quad H_{1} \neq H_{2} .
$$

To find the roots of Eq.(2.6), the algorithm below is used for each $x$ between 0 and $L$ :

$$
\text { if : } \quad 0<\frac{d p}{d x}<\frac{8 \mu U}{H_{1}^{2}} \quad \text { then } \quad H_{2}(x)=\frac{-H_{1}(x)}{2}+\sqrt{\frac{6 \mu U}{\frac{d p}{d x}}-\frac{3}{4} H_{1}^{2}(x)}
$$

else:

$$
H_{2}(x)=H_{1}(x)
$$

Equation (2.7) leads to the following condition regarding the unicity of the film thickness:

$$
\frac{d p}{d x} \geq \frac{8 \mu U}{H_{l}^{2}} \quad \text { or } \quad \frac{d p}{d x} \leq 0 \quad \text { for each } \quad x \quad \text { between } \quad 0 \quad \text { and } \quad L .
$$


It is important to note that when the condition (2.9) is not verified, all the roots film thickness $\mathrm{H}_{2}$ could be combined to the initial film thickness $H_{1}$, and generate a new film thickness $h(x)$ with the equation:

$$
h(x)=\alpha(x) H_{1}(x)+(1-\alpha(x)) H_{2}(x)
$$

where: $\alpha$ is an entire coefficient of geometry depending on the coordinate $x$ and is equal to $l$ or 0 , and $H_{l}(x)$ is the initial film thickness and $H_{2}(x)$ are the roots film thickness.

The parameter $\alpha(x)$ defines a new surface generating the same hydrodynamic pressure. Later in this paper, an investigation is performed to demonstrate how far the pressure is similar by considering the cavitation effect.

\section{Applications}

\subsection{Different bearing geometry: without cavitation effect}

To explain this new theory in detail, an example of a commonly known bearing "sloping surface" is studied as described in Fig.4.

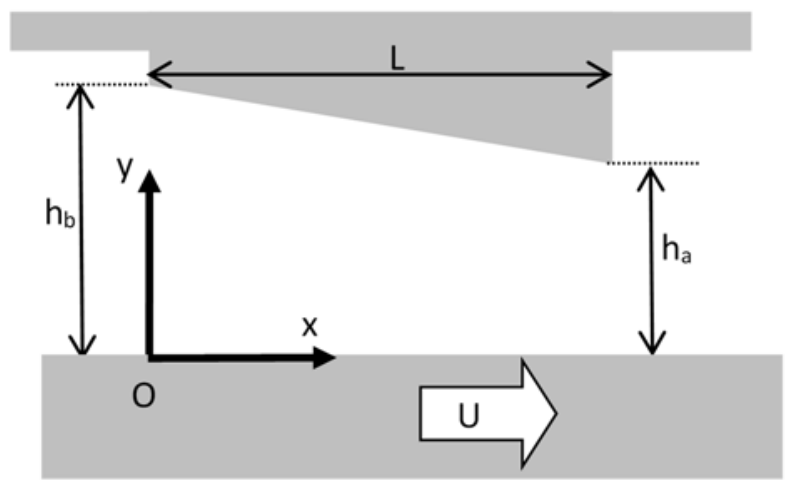

Fig.4. The bearing geometry "sloping surface".

Figure 4 shows the flow between two non-parallel flat plates of infinite width along $(O z)$ and height difference at the ends $h_{a}$ and $h_{b}$ with a length $L$. The lower plate is driven by a uniform translation movement of speed $U$, the upper plate is stationary and inclined in the $(x O y)$ plane by a very small constant angle.

By assuming the following data: $L=20 \mathrm{~mm}, h_{b}=0.008 \mathrm{~mm}, h_{a}=0.002 \mathrm{~mm}, \mu=0.01 \mathrm{~Pa} \cdot \mathrm{s}$, $U=200 \mathrm{~mm} / \mathrm{s}$ the initial film thickness, in this case, is given by the following equation:

$$
H_{l}(x)=h_{a}+\frac{\left(h_{b}-h_{a}\right)}{L}(L-x)
$$

With those operational conditions, the current bearing geometry generates the hydrodynamic pressure shown in Fig.5. A comparison between the finite difference method used in the previous section and the analytical result was performed and the numerical algorithm was easily validated. 


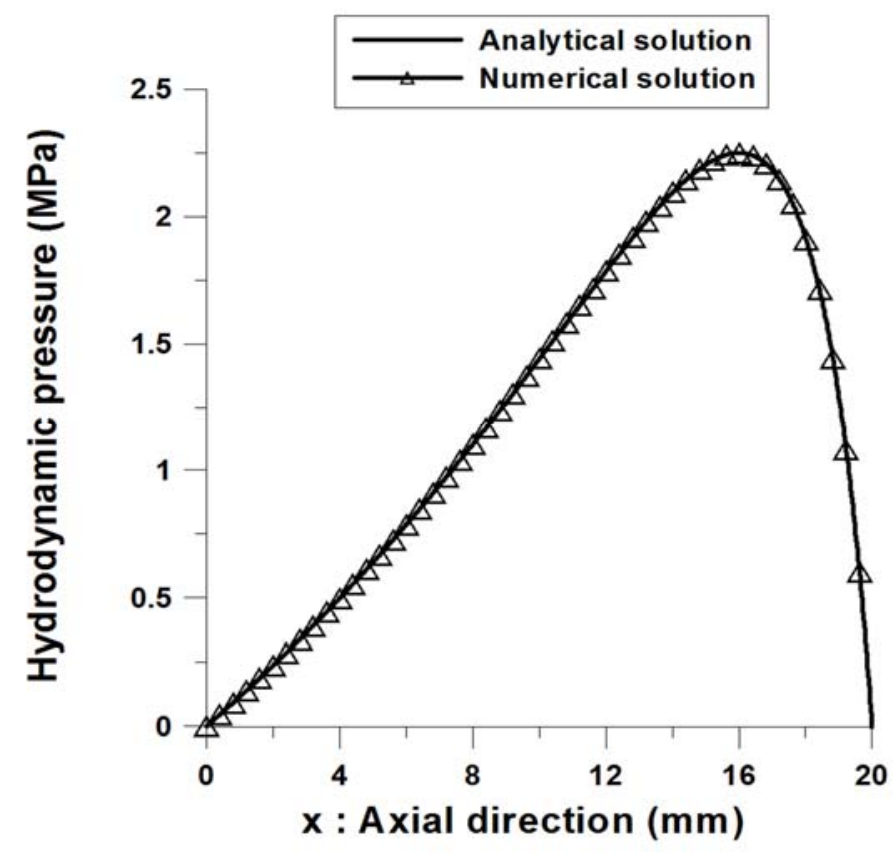

Fig.5. Hydrodynamic pressure for an inclined surface bearing.

Figure 6 proposes different configurations: $H_{1}$ and $H_{2}$ to design an appropriate geometry for the load support, keeping the same hydrodynamic lifting force and the same flow rate. Indeed, for illustrative purposes, all the shapes shown in Fig.7. could generate the same performance by only combining the initial and second film thickness within the parameter $\alpha(x)$ in Eq.(2.10).
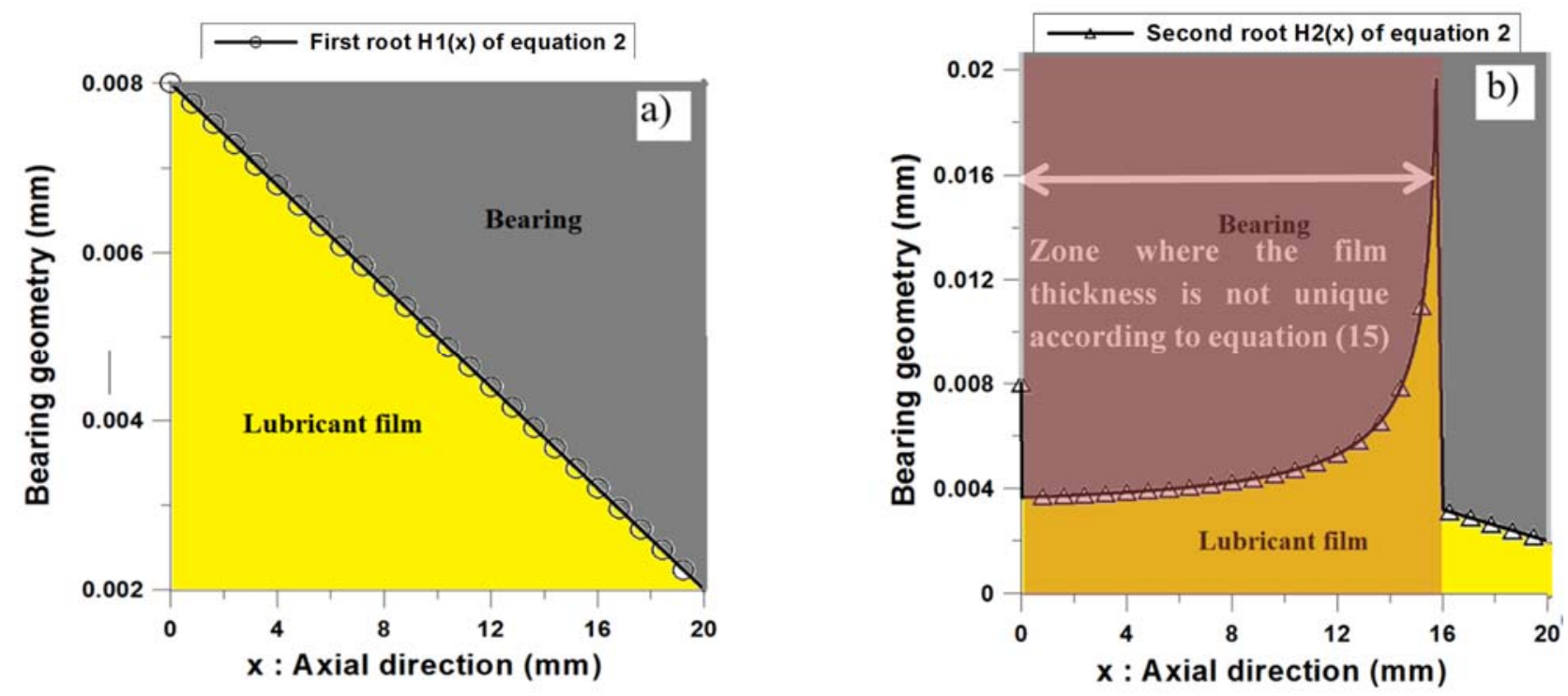

Fig.6. The film thicknesses roots: a) the initial film thickness $H_{1}(x)$, b) the second film thickness computed from Eqs (2.7) and (2.8). 
It is relevant to confirm that with all the three bearing surface shapes $\# 1, \# 2$, and $\# 3$, the same hydrodynamic pressure is generated as shown in Fig.8., and the same flow rate as demonstrated in Fig.9.
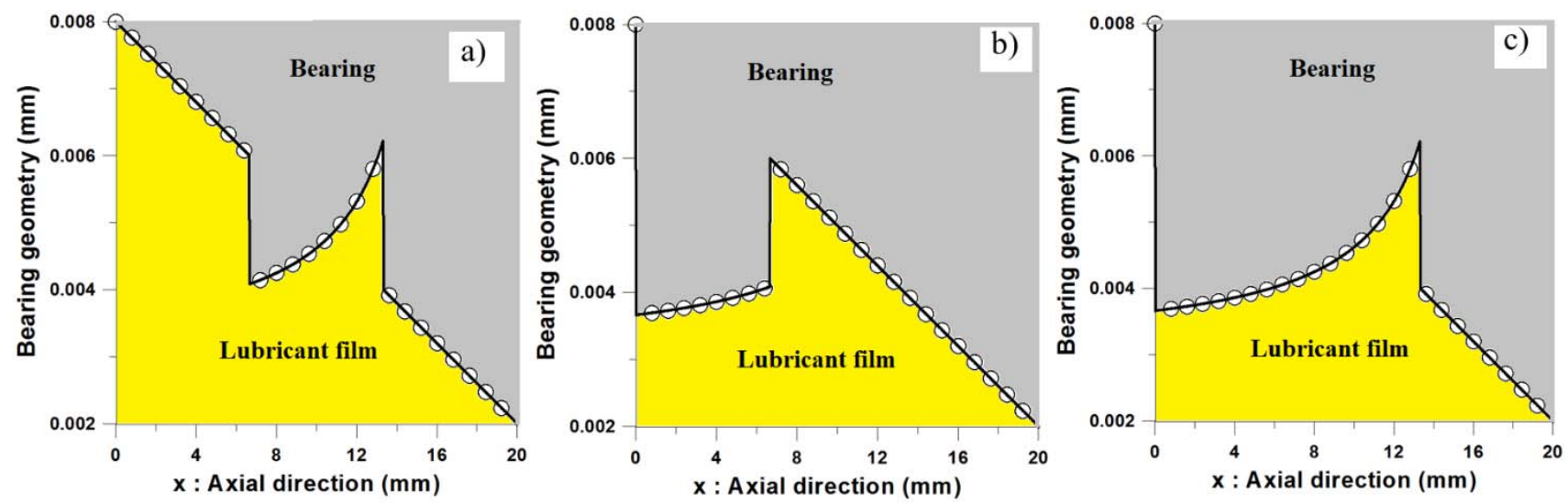

Fig.7. Examples of different bearing shape, a) shape \#1, b) shape \#2, c) shape \#3.

It is also important to note that the frictional force difference between the three bearing surfaces is slightly regular. Thus with shape \#1, the friction force per length is about $12.98 \mathrm{~N} / \mathrm{m}$, shape \#2 is $13.16 \mathrm{~N} / \mathrm{m}$, and $13.17 \mathrm{~N} / \mathrm{m}$ for shape \#3.

Thus the designers need to perform several iterations by changing geometry to maintain the same lifting force and flow rate and minimizing the viscous friction effect.

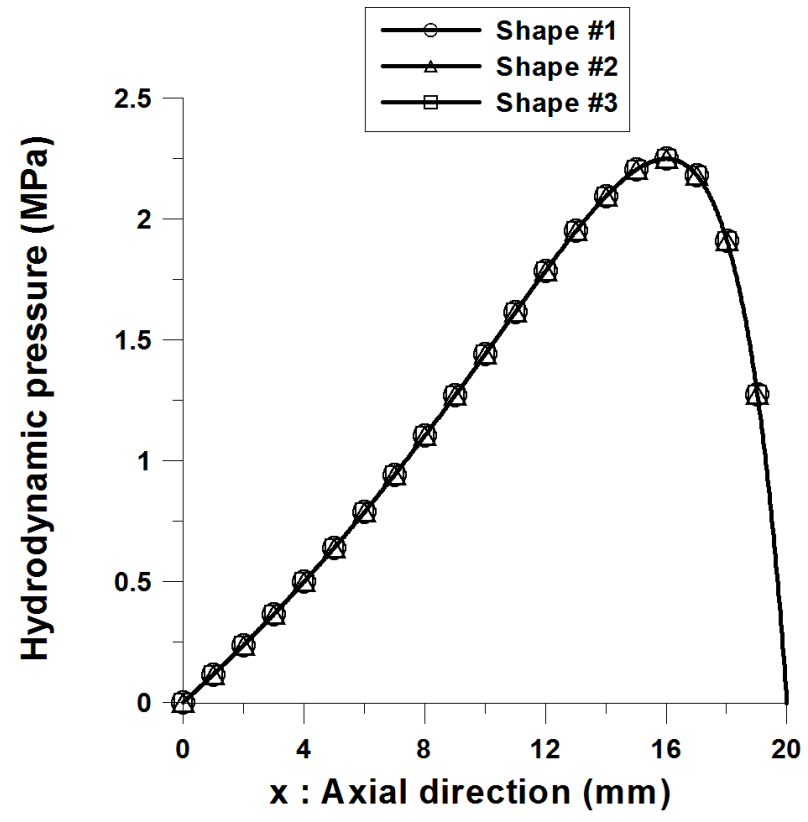

Fig.8. Hydrodynamic pressure with different bearing geometry.

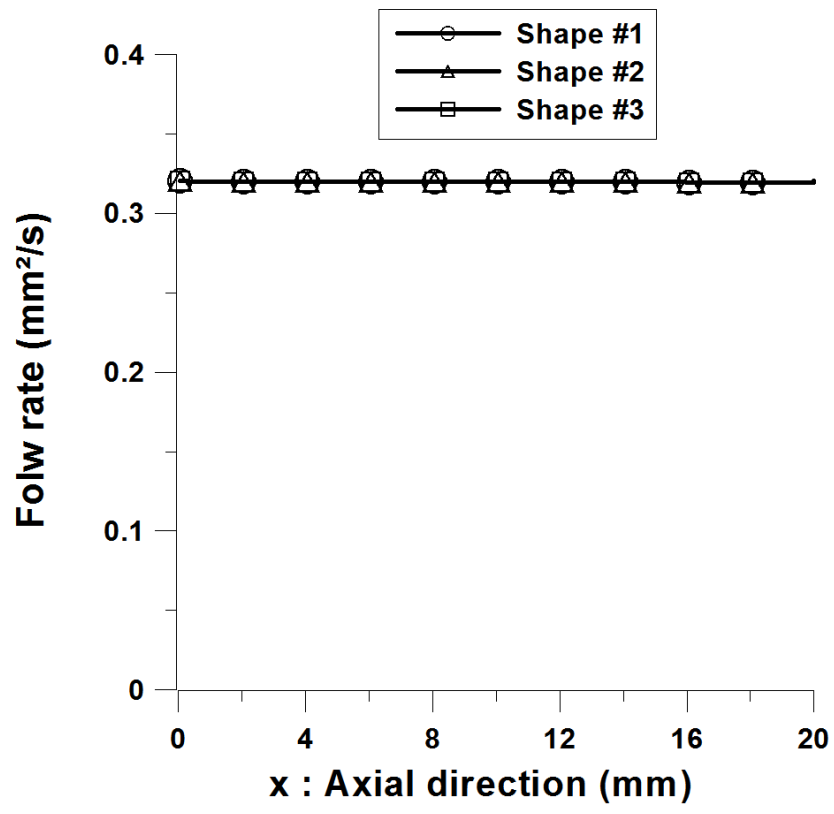

Fig.9. Flow rate conservation with different bearing geometry. 


\subsection{Micro-textured surfaces}

After confirming the existence of different film thicknesses generating similar hydrodynamic pressure in the case of the bearing with macro shaped geometry, this section presents a second application in the lubricated contact by considering micro-textured surfaces.

Table 2. Geometry and operating conditions of the microtextured surface.

\begin{tabular}{|l|l|}
\hline Parameter & Numerical value \\
\hline \multirow{2}{*}{ Upper surface length } & $L=100 \mathrm{~mm}$ (Non-cavitated case) \\
\cline { 2 - 2 } & $L=76 \mathrm{~mm}$ (Cavitated case) \\
\hline Inlet length of cell texture & $a=4 \mathrm{~mm}$ \\
\hline The first length of cell texture & $b=6 \mathrm{~mm}$ \\
\hline The second length of cell texture & $c=10 \mathrm{~mm}$ \\
\hline Outlet length of cell texture & $d=24 \mathrm{~mm}$ (Non-cavitated case) \\
\cline { 2 - 2 } & $d=0 \mathrm{~mm}$ (Cavitated case) \\
\hline Texture depth & $h_{d}=5 \mathrm{micron}$ \\
\hline Separating space film thickness & $h_{0}=5 \mathrm{micron}$ \\
\hline Texture density & $N=4$ \\
\hline Velocity & $U=1 \mathrm{~m} / \mathrm{s}$ \\
\hline Dynamic viscosity & $m=0.01 \mathrm{~Pa} . \mathrm{s}$ \\
\hline Ambient pressure & $p_{0}=0.1 \mathrm{MPa}$ \\
\hline Cavitation pressure & $p_{\text {cav }}=0 \mathrm{MPa}$ \\
\hline Node number & $N=1000 \mathrm{Nodes}$ \\
\hline
\end{tabular}

This part aims to find all micro-textured surfaces providing the same lifting force by considering the phenomena resulting from the convergent-divergent domain, especially the cavitation effect.

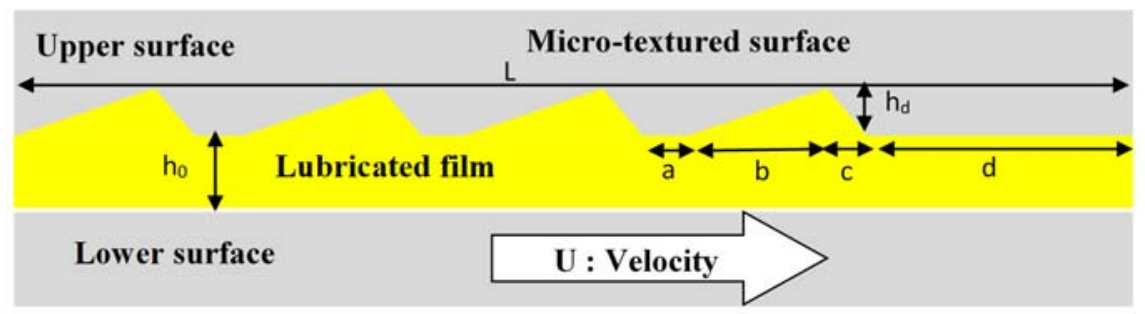

Fig.10. The micro-textured bearing geometry.

Figure 10 illustrates the considered micro-textured surface with the operational parameters presented in Tab.2.

\subsubsection{First case: $L=100 \mathrm{~mm}, d=24 \mathrm{~mm}$ (without cavitation effect)}

A micro-textured surface is considered with an initial film thickness $H_{1}(x)$ given in Fig.10. This study is performed throughout three steps: 1$)$ computing the hydrodynamic pressure $p(x)$ with the guessed initial film thickness $H_{1}(x)$ and resolving the Reynolds equation (1.1), 2) using the Eqs (2.7) and (2.8) to find 
the roots film thickness $\left.H_{2}(x), 3\right)$ comparing hydrodynamic pressures $p(x)$ corresponding to the film thickness $H_{1}(x)$ and $H_{2}(x)$ with the modified Reynolds equation.

Step 1: The hydrodynamic pressure corresponding to the initial film thickness $H_{1}(x)$ :

According to the discretized equation (1.6), the Reynolds equation is resolved. Figure 11 shows the hydrodynamic pressure $p(x)$ corresponding to the initial film thickness $H_{l}(x)$.



Fig.11. Hydrodynamic pressure according to the initial film thickness $H_{1}(x)$.

Step 2: The second film thickness roots $H_{2}(x)$ :

By using Eqs (2.8) and (2.9), the second film thickness $H_{2}(x)$ is computed. Figure 12 shows the big difference between the first film thickness $H_{1}(x)$ and $H_{2}(x)$. It is important to underline that both film thicknesses generate the same hydrodynamic pressure $p(x)$.
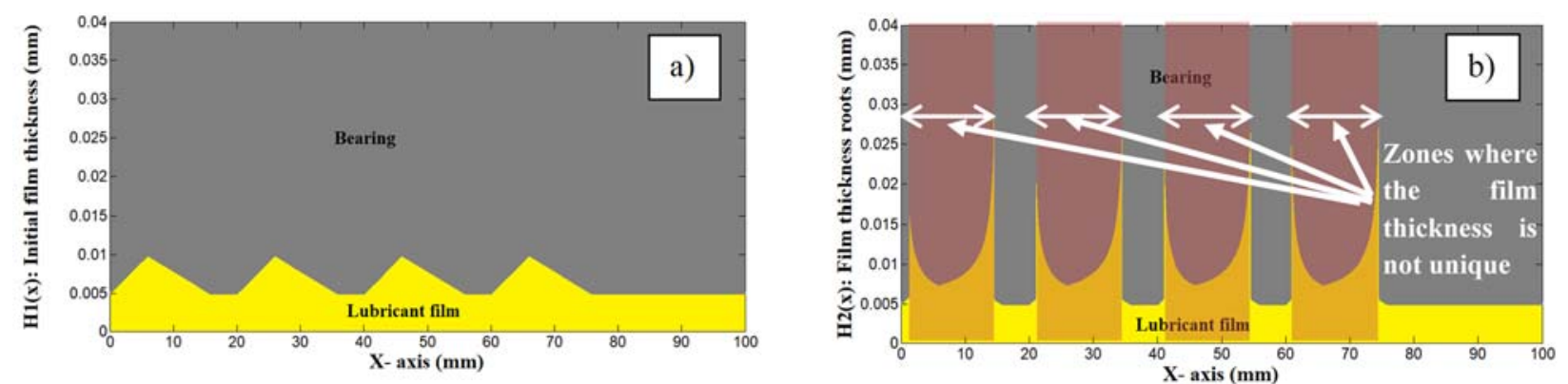

Fig.12. The film thicknesses roots without the cavitation effect: a) the initial film thickness $H_{l}(x)$, b) the second film thickness computed from Eqs (2.7) and (2.8).

Step 3: Comparing the hydrodynamic pressure for each film thickness

According to numerical results of step 2, Fig.13. confirms the similar hydrodynamic pressure provided by different film thicknesses $H_{1}(x)$ and $H_{2}(x)$. 


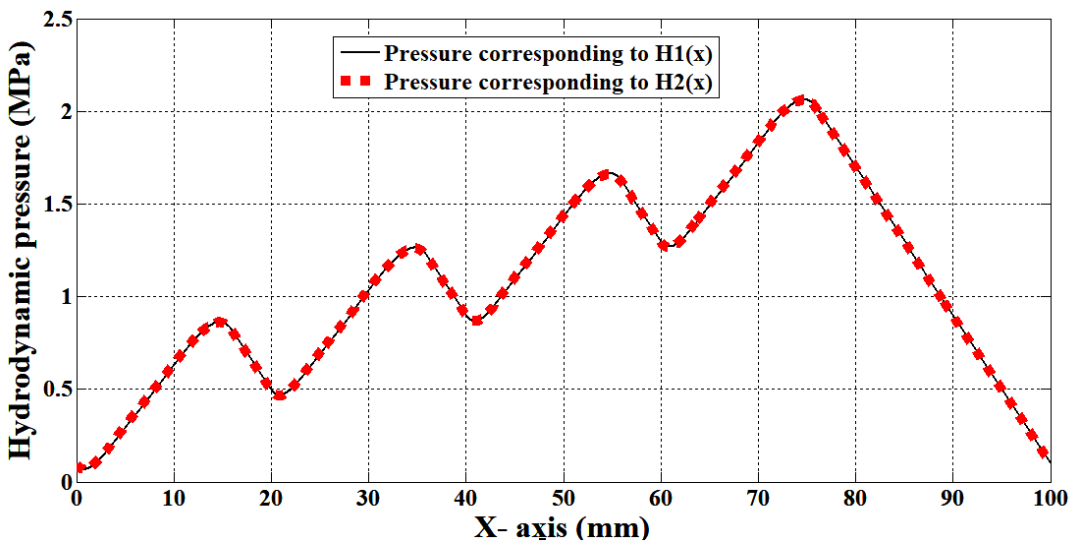

Fig.13. Hydrodynamic pressure for different micro-textured surfaces without cavitation.

\subsubsection{Second case: $L=76 \mathrm{~mm}, d=0 \mathrm{~mm}$ (with cavitation effect)}

To investigate the cavitation effect on the second film thickness $H_{2}(x)$, this section aims to study microtextured surface generating cavitated zone with $L=76 \mathrm{~mm}$ and $d=0 \mathrm{~mm}$ as shown in Fig.10. This analysis is performed throughout three steps: 1) resolving the Reynolds equation (1.1) to find the hydrodynamic pressure $p(x)$ with the initial film thickness $\left.H_{l}(x), 2\right)$ using Eqs (2.7) and (2.8) to compute the second film thickness $\left.H_{2}(x), 3\right)$ comparing hydrodynamic pressures $p(x)$ corresponding to the film thickness $H_{1}(x)$ and $H_{2}(x)$.

Step 1: The hydrodynamic pressure corresponding to the initial film thickness $H_{1}(x)$, with the Gümbel [11] cavitation condition.

The Reynolds equation is resolved by considering the Gümbel conditions [11] given in Eq.(1.5). Figure 11 shows the hydrodynamic pressure $p(x)$ corresponding to the initial film thickness $H_{l}(x)$, where the existence of the cavitated zones is highlighted.

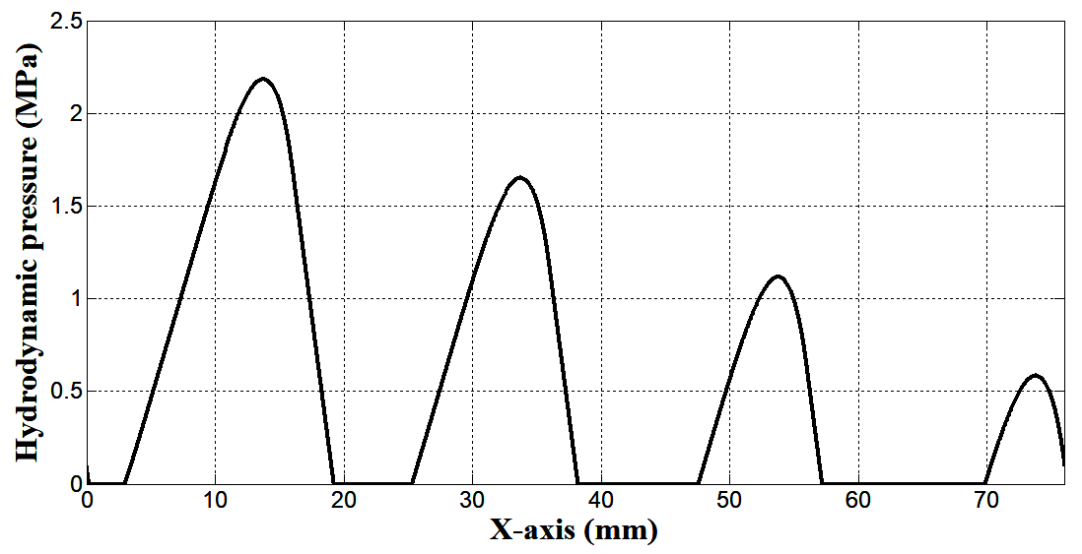

Fig.14. Hydrodynamic pressure with the initial film thickness $H_{l}(x)$.

Step 2: The film thickness roots $\mathrm{H}_{2}(x)$ :

The second film thickness $\mathrm{H}_{2}(x)$ is computed from Eqs (2.8) and (2.9) by using the hydrodynamic pressure corresponding to the initial film thickness $H_{l}(x)$. Figure 15 demonstrates the impact of the cavitation 
on the second film thickness $H_{2}(x)$. Indeed, the initial film thickness $H_{1}(x)$ is nearly equal to the second $H_{2}(x)$ except in the vicinity of the border between the cavitated and non-cavitated zone.
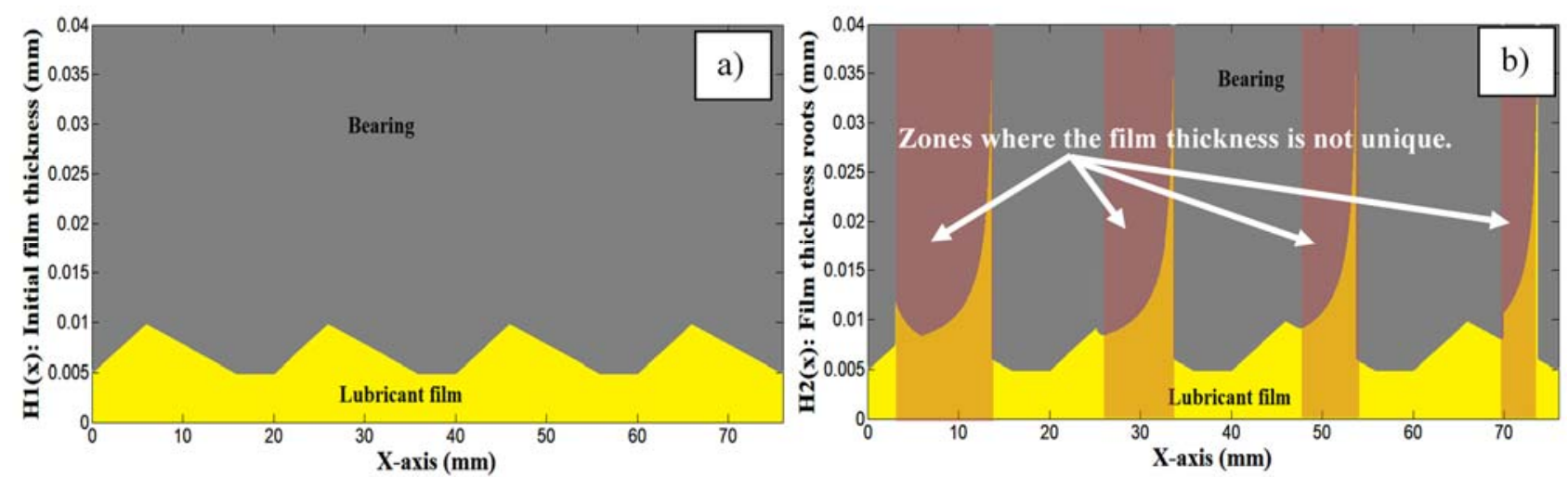

Fig.15. The film thicknesses roots with cavitation effect: a) the initial film thickness $H_{l}(x)$, b) the second film thickness computed from equations (2.7) and (2.8).

Step 3: Comparing the hydrodynamic pressure for each film thickness (with the modified Reynolds equation) To compare the hydrodynamic pressure generated with the film thicknesses: the initial $H_{l}(x)$ and the second $\mathrm{H}_{2}(x)$, the modified Reynolds equation was resolved of both thicknesses by considering the mass conservation instead of the Gümbel model [11] according to Eq.(1.5).

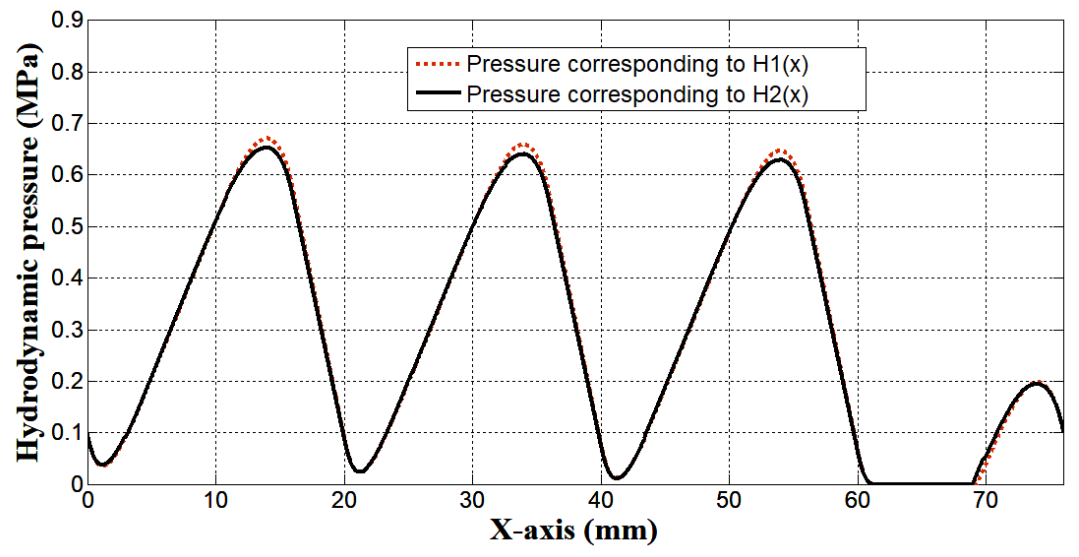

Fig.16. Comparison of the hydrodynamic pressure provided by the initial film thickness $H_{l}(x)$ and the second film thickness $H_{2}(x)$.

Figure 16 confirms a slight difference in the hydrodynamic pressure corresponding to the film thicknesses $H_{1}(x)$ and $H_{2}(x)$, despite the big difference with the hydrodynamic pressure given by the initial film thickness $H_{1}(x)$ computed according to the Gümbel model [11]. Therefore, it is also relevant to underline that the same initial film thickness $H_{1}(x)$ gives two different hydrodynamic pressures with mass conservation according to the modified Reynolds equation (1.6) and with the Gümbel cavitation model (1.5). 


\subsubsection{Effect of separating space film thickness: $h_{0}$.}

It is well known that the gap that separates lubricated surfaces affects significantly the hydrodynamic pressure. Thus, this section aims to prove the effectiveness of such an operational parameter on the second film thickness by considering the cavitation effect.

To perform this investigation, a similar micro-textured surface is considered with $L=76 \mathrm{~mm}$ and $d=0 \mathrm{~mm}$ as described in Fig.10 and three different separating gaps are designed as follows: Case \#1 with $h_{0}=5$ microns, Case \#2 with $h_{0}=1$ micron and Case \#3 with $h_{0}=10$ microns .
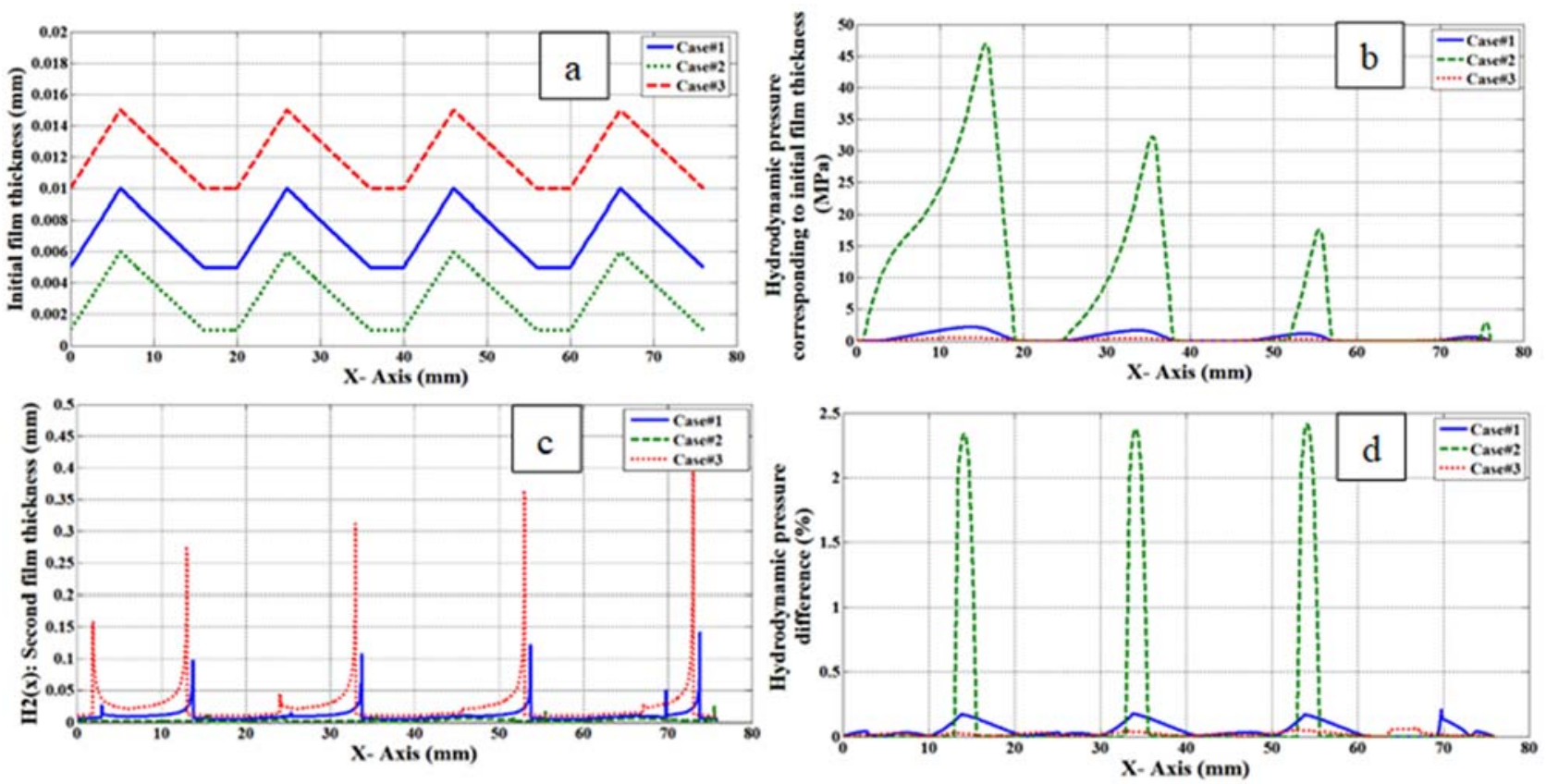

Fig.17. Comparison of three separating gaps: a) initial film thickness $H_{l}(x)$, b) hydrodynamic pressure corresponding to $H_{1}(x)$ according to the Gümbel model, c) second film thickness $H_{2}(x)$, d) difference between hydrodynamic pressure computed from $H_{1}(x)$ and $H_{2}(x)$ according to the modified Reynolds equation.

Figure 17 shows the impact of the gap between lubricated surfaces. As long as surfaces are close and the hydrodynamic pressure is high, the deviation between the hydrodynamic pressure produced by the second thickness is large compared to that generated by the initial thickness. Although the differences in pressure do not exceed $2 \%$, the deviation maximum is located at the the border of cavitation zone and to the non-cavitated zone. Consequently, the proposed method for generating a second film thickness is applicable for any spacing gap between lubricated surfaces.

\subsection{Rough surfaces}

In general, the roughness surface is the relevant parameter that significantly affects the lubricated contact. Previous works have demonstrated that the arithmetic roughness $R a$, the standard deviation $\sigma$, the kurtosis and skewness parameters $K u$, and $S k$, respectively, define the topography surface capacity to characterize tribological behavior of surfaces. 
One of the pillar methods to find the hydrodynamic pressure for each rough surface is based on the stochastical approach with Patir and Cheng flow factors [7]. This stochastic method defines pressure as a function of the averaged film thicknesses ratio to the standard deviation roughness $h_{0} / \sigma$.

The main subject of this section is to prove that different surface topography parameters: $R a, K u$, and $S k$ could generate the same hydrodynamic pressure. It is also very relevant to demonstrate that with different standard deviation $\sigma$ the hydrodynamic pressure could be maintained, thus the adimensional film thickness $h_{0} / \sigma$ is not sufficient to define the hydrodynamic or elasto-hydrodynamic regime.

Table 3 gives the initial film thickness (respectively, the initial surface roughness), and the second film thickness is given by Eqs (2.7) and (2.8), with operational parameters as described in Tab.1. with a lubricated length $L=76 \mathrm{~mm}$ and separating gap $h_{0}=1$ micron .

The standard deviation ( so-called Root Mean Square RMS) $\sigma$, the arithmetic roughness $R a$, kurtosis $K u$, and skewness $S k$ are computed for each film thickness $H$ as follows:

$$
\begin{aligned}
& R(x)=H(x)-h_{0}, \\
& \bar{R}=\frac{1}{N} \sum_{i=1}^{N} R_{i}=0 \quad \text { (Assumption of randomized signal), } \\
& \sigma=\sqrt{\frac{1}{N} \sum_{i=1}^{N}\left(R_{i}-\bar{R}\right)^{2}} \\
& S k=\frac{1}{\sigma^{3} N} \sum_{i=1}^{N}\left(R_{i}-\bar{R}\right)^{3} \\
& K u=\frac{1}{\sigma^{4} N} \sum_{i=1}^{N}\left(R_{i}-\bar{R}\right)^{4}
\end{aligned}
$$

where: $R$ is the roughness function represented in the realistic case with a randomized signal, and $N$ is the total node number.

Table 3. Initial and second roughnesses corresponding to film thickness $H_{l}(x)$ and $H_{2}(x)$, respectively.

\begin{tabular}{|l|c|c|c|c|}
\hline \multirow{2}{*}{ Surface code } & \multicolumn{4}{|c|}{ Surface topography parameters } \\
\cline { 2 - 5 } & $\begin{array}{c}\text { Arithmetic } \\
\text { roughness: } R a\end{array}$ & $\begin{array}{c}\text { Standard } \\
\text { deviation: } \sigma\end{array}$ & $\begin{array}{c}\text { Kurtosis: } \\
\mathrm{Ku}\end{array}$ & $\begin{array}{c}\text { Skewness: } \\
\mathrm{Sk}\end{array}$ \\
\hline Initial film thickness: Rough\#1 & $1,00 \times 10^{-3} \mathrm{~mm}$ & $338,08 \times 10^{-6} \mathrm{~mm}$ & 132,84 & 34,82 \\
\hline Second film thickness: Rough\#2 & $1,26 \times 10^{-3} \mathrm{~mm}$ & $1,53 \times 10^{-3} \mathrm{~mm}$ & 96,53 & 10,22 \\
\hline
\end{tabular}

Figure 18 shows numerical simulation by considering randomized surface as an initial surface roughness with a separating gap $h_{0}=1$ micron, with the operational parameters in Tab.2. The second film thickness $H_{2}(x)$ is deduced from Eqs (2.7) and (2.8) as given in Fig.18a. Before computing the film thickness 
$H_{2}(x)$, a post-processing step is important: computing the hydrodynamic pressure generated by the initial film thickness $H_{1}(x)$ throughout resolving equation (1.1) and using the Gümbel cavitation model [11].

To compare the accuracy of simulations, the hydrodynamic pressure produced by both film thicknesses $H_{1}(x)$ and $H_{2}(x)$ is computed by the modified Reynolds equation (1.6).
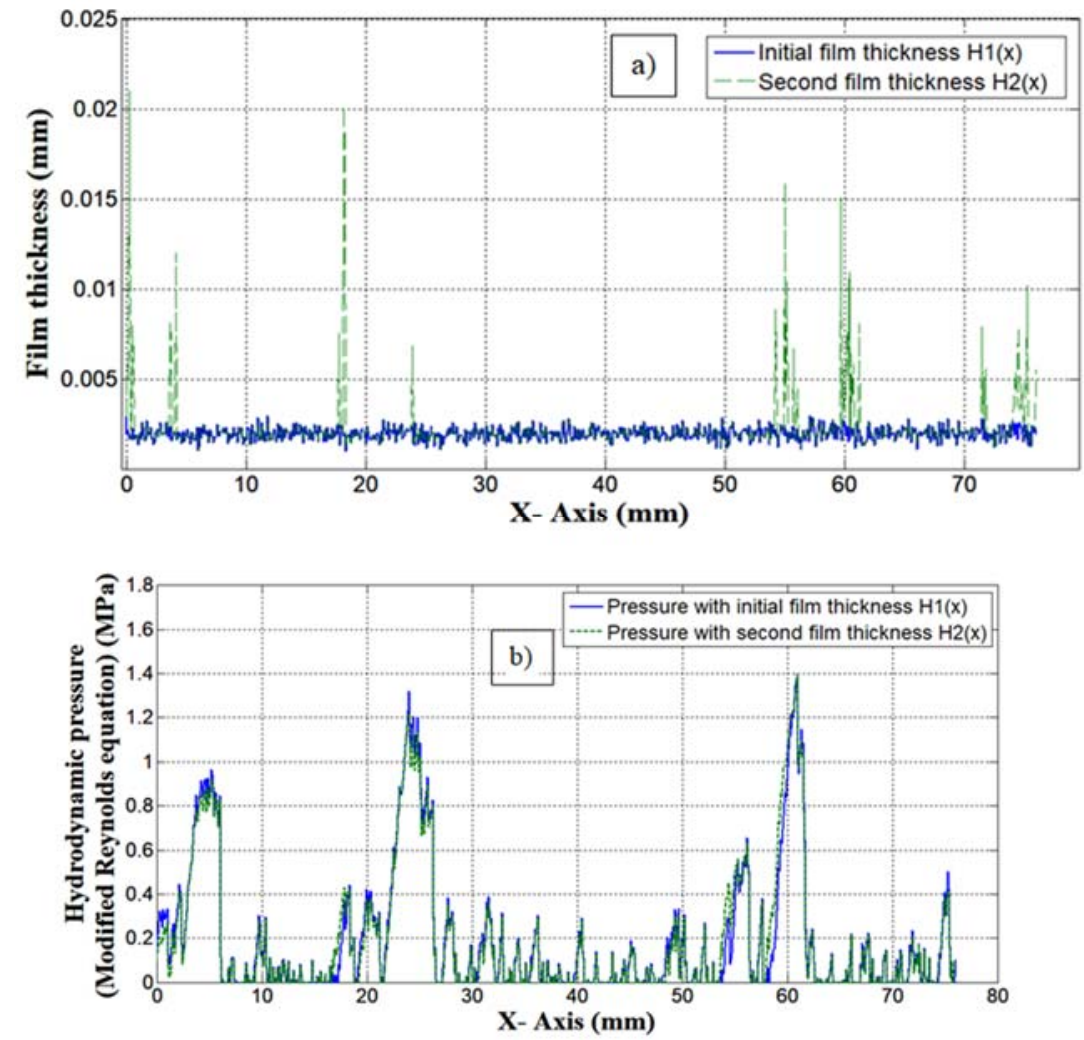

Fig.18. Comparison of hydrodynamic pressure values for different film thicknesses: a) initial film thickness $H_{1}(x)$, b) hydrodynamic pressure computed from $H_{1}(x)$ and $H_{2}(x)$ by the modified Reynolds equation.

Figure $18 \mathrm{~b}$ confirms that the pressure has slight differences in spite of the significant differences between surface topography, especially the Root Mean Square (RMS) $\sigma$. Thereby, it is important to note that the ratio $h_{0} / \sigma$ is not sufficient to determine the lubrication regime, thereby the deficiency of this ratio to define the lubrication regime as commonly known from the Patir and Cheng theory.

\section{Conclusions}

In this paper, the existence of several film thicknesses that provide the same hydrodynamic pressure by considering the cavitation effect was investigated throughout three depending steps:

Step 1: With the operational conditions and an initial film thickness $H_{l}(x)$, the hydrodynamic pressure is computed from the Reynolds equation and using the Gümbel cavitation condition.

Step 2: By using the hydrodynamic pressure of step 1 and under a specific pressure gradient condition, a second film thicknesses $H_{2}(x)$ is found with the presented new algorithm. 
Step 3: Comparing the hydrodynamic pressure accuracy of both thicknesses $H_{1}(x)$ and $H_{2}(x)$ computed from the mass conservation equation with the modified Reynolds equation.

This new approach was applied for different lubricated surfaces:

- Macro-shaped surfaces: by considering initial bearing surfaces, several surfaces have been proposed that give the same hydrodynamic pressure. It was also demonstrated that with different bearing macro-shapes the frictional force varies slightly and the flow rate is regular.

- Micro-textured surfaces without cavitation: this case concerns a specific geometry condition of the surfaces that avoid the cavitation effect. By using the presented method, the initial and the second film thicknesses generate the same hydrodynamic pressure computed from the modified Reynolds equation.

- Micro-textured surfaces with the cavitation effect: a specific geometry condition was considered to produce cavitation zones within the lubricated contact. $0.1 \%$ is a difference gap between the pressure obtained by resolving the modified Reynolds equation with both film thicknesses.

- Rough surface: a randomized surface was considered and a second film thickness $\mathrm{H}_{2}(x)$ corresponding to a new roughness generating the same hydrodynamic pressure was computed. It was confirmed also that the pressure has a slight difference in spite of the significant differences between the Root Mean Square (RMS) $\sigma$.

The effect of the separating gap between the lubricated surfaces was also investigated and the second film thickness was found. The impact of this separation is about $2 \%$ by comparing the hydrodynamic pressure for both thicknesses computed from the modified Reynolds equation.

This paper underlines that the ratio $h_{0} / \sigma$ is not sufficient to determine the lubrication regime as commonly known from the Patir and Cheng theory.

It must be recalled that this method is validated without considering the dry contact phenomenon. This work opens new tracks to be investigated to enable design of lubricated contact with any pressure distribution coupled with the surface flexibility.

\section{Nomenclature}






\section{References}

[1] Fatu A., Maspeyrot P. and Hajjam M. (2011): Wall slip effects in (elasto) hydrodynamic journal bearings.- Tribology International, vol.44, pp.868-877.

[2] Gherca A.R., Maspeyrot P., Hajjam M. and Fatu, A. (2013): Influence of texture geometry on the hydrodynamic performances of parallel bearings.- Tribology Transactions, vol.56, pp.321-332.

[3] Kanters A.F.C., Verest J.F.M. and Visscher M. (1990): On reciprocating elastomeric seals: calculation of film thicknesses using the inverse hydrodynamic lubrication theory.- Tribology Transactions, vol.33, pp.301-306.

[4] Nikas G.K. and Sayles R.S. (2006): Study of leakage and friction of flexible seals for steady motion via a numerical approximation method.- Tribology International, vol.39, pp.921-936.

[5] Fatu A. and Hajjam M. (2011): Numerical modelling of hydraulic seals by inverse lubrication theory.- Proc. IMechE, Part J: J. Engineering Tribology, vol.225, pp.1159-1173.

[6] Crudu M., Fatu A., Hajjam M. and Cristescu C. (2013): Numerical and experimental study of reciprocating rod seals including surface roughness effects.- Sealing Technology, vol.6, pp.8-11.

[7] Patir N. and Cheng H.S. (1978): An average flow model for determining effects of three-dimensional roughness on partial hydrodynamic lubrication.- ASLE Trans., pp.12-17.

[8] Shen D. and Salant R.F. (2006): A transient mixed lubrication model of rotary lip seal with a rough shaft.- STLE Tribology Transactions, vol.49, pp.621-634.

[9] Fowell M., Olver A.V., Gosman A.D., Spikes H.A. and Pegg I. (2007): Entrainment and inlet suction: two mechanisms of hydrodynamic lubrication in textured bearings.- ASME Journal of Tribology, vol.129, pp.336-345.

[10] Fowell M.T., Medina S., Olver A.V., Spikes H.A. and Pegg, I.G. (2013): Parametric study of texturing in convergent bearings.- Tribology International, vol.52, pp.7-16.

[11] Gumbel L. (1921): Verglieich der Ergebnisse der rechnerischen Behaudlung des lagerschmierungs problem mit neuren Versuchsergebnissen.- Monatsblätter d. Berlin, Bezirk V.D.I., pp.125-128.

[12] Lahjouji I., El Gadari M., El Fahime B. and Radouani M. (2017): Effect of relative velocity between rough surfaces: hydrodynamic lubrication of rotary lip seal.- Int J Appl Mech Eng, vol.22, No.2, pp.321-332.

Received: March 7, 2021

Revised: July 14, 2021 